\title{
EFECTO DE DISTINTOS NIVELES DE PODA Y REPOSICIÓN HÍDRICA SOBRE EL CRECIMIENTO VEGETATIVO, RENDIMIENTO Y COMPOSICIÓN DE BAYAS EN VIDES CV. CABERNET SAUVIGNON
}

\author{
Effect of different levels of pruning and water application on vegetative growth, \\ yield and berry composition in grapes cv. Cabernet Sauvignon
}

\author{
Samuel Ortega-Farias ${ }^{1 *}$, Rodrigo Salazar Mejías ${ }^{1}$, y Yerko Moreno Simunovic ${ }^{2}$
}

\section{A B S T R A C T}

An experiment was carried out to evaluate the effects of different levels of pruning and water application on vegetative growth, yield and berry composition of grapevines (Vitis vinifera L.), located in Pencahue Valley, Maule Region, Chile ( $35^{\circ} 22^{\prime}$ S lat; $71^{\circ} 47^{\prime} \mathrm{W}$ long), during the 2003-2004 and 2004-2005 growing seasons. The cultivar used was a 10 yr-old Cabernet Sauvignon, drip irrigated and trained on a vertical shoot positioned system. Pruning levels were 12, 18 and 24 buds per plant. Irrigation levels applied were 40,70 and $100 \%$ of real evapotranspiration of the vineyard (ETv) from setting to harvest. Results showed that increasing pruning severity resulted in a decrease in the Ravaz index from 4.3 to 2.9 in the first season, and a severe reduction of yield (between 41 and 53\%) in both seasons. Only in the second season the reduction in water application decreased the yield from 3.0 to $1.8 \mathrm{~kg} \mathrm{pl}^{-1}$ and increased the total anthocyanin content in berries from 1290 to 1520 $\mathrm{mg} \mathrm{L}^{-1}$. There was no significant interaction between pruning and irrigation levels for any of the seasons under evaluation.

Key words: pruning, irrigation levels, vegetative growth, yield, berry composition, Cabernet Sauvignon, Vitis vinifera.

\section{R E S U M E N}

\begin{abstract}
Se realizó un experimento para evaluar el efecto de distintos niveles de poda y reposición hídrica sobre el crecimiento vegetativo, rendimiento y composición de bayas en un viñedo (Vitis vinifera L.) ubicado en el Valle de Pencahue, Región del Maule, Chile (35 $22^{\prime}$ lat. Sur; $71^{\circ} 47^{\prime}$ long. Oeste), durante las temporadas de crecimiento 2003-2004 y 2004-2005. Se usó un cultivar Cabernet Sauvignon de 10 años de edad, regado por goteo y conducido en espaldera vertical simple. Los distintos niveles de poda fueron 12, 18 y 24 yemas por planta. Los niveles de riego consistieron en la reposición del 40, 70 y $100 \%$ de la evapotranspiración real de la vid (ETv) aplicado durante el período de cuaja a cosecha. Los resultados mostraron que al incrementar la severidad de poda se obtuvo una disminución del índice de Ravaz desde 4,3 a 2,9 en la primera temporada y una reducción severa del rendimiento (entre un 41 y $53 \%$ ) en ambas temporadas. Sólo en la segunda temporada, la reducción en la reposición hídrica disminuyó en forma significativa el rendimiento desde 3,0 a $1,8 \mathrm{~kg} \mathrm{pl}^{-1}$ e incrementó el contenido de antocianas totales desde 1.290 a 1.520 $\mathrm{mg} \mathrm{L}^{-1}$ en las bayas. En ninguna de las dos temporadas de evaluación se registraron interacciones positivas importantes entre los factores poda y riego.
\end{abstract}

Palabras clave: poda, reposición hídrica, crecimiento vegetativo, rendimiento, composición de bayas, $\mathrm{Ca}-$ bernet Sauvignon, Vitis vinifera.

\footnotetext{
${ }^{1}$ Universidad de Talca, Facultad de Ciencias Agrarias, Centro de Investigación y Transferencia en Riego y Agroclimatología (CITRA), Casilla 747, Talca, Chile.

E-mail: sortega@utalca.cl_*utor para correspondencia.

${ }^{2}$ Universidad de Talca, Facultad de Ciencias Agrarias, Centro Tecnológico de la Vid y el Vino (CTVV), Casilla 747, Talca, Chile. Recibido: 2 de octubre de 2006. $\quad$ Aprobado: 27 de diciembre de 2006.
} 


\section{INTRODUCCIÓN}

Entre las prácticas tendientes a mejorar la calidad final de mostos y vinos está el control del vigor de las plantas mediante labores culturales de poda (por ejemplo, ajustando el número de yemas por metro lineal) y riego (estrategias de riego deficitario controlado) (Ferreira et al., 1998; Acevedo et al., 2004; Ortega-Farias et al., 2004a). Estas labores pueden afectar el comportamiento de la vid en forma importante. Por ejemplo, determinar una muy baja carga de yemas podría implicar una disminución en la producción, con un consecuente aumento del diámetro de los brotes y un incremento generalizado del vigor, situación que podría acentuarse aún más con el corrimiento de racimos y un desequilibrio de la planta, donde la producción final de los vinos se vería afectada negativamente.

Recíprocamente, una excesiva carga de yemas conduce a una mayor densidad de brotes, carga frutal y área foliar, aumentando la competencia entre la fruta y los brotes, lo que ocasionaría una maduración desuniforme y una baja calidad de la fruta, además de una lignificación insuficiente y un debilitamiento de la planta (Reynier, 1989). Incrementos del ácido málico, potasio, y pH de las bayas están asociados al vigor excesivo, al igual que una disminución del color y sólidos solubles ( ${ }^{\circ}$ Brix), reduciendo significativamente la calidad del vino (Pszczólkowski y Bordeu, 1984). Además, la relación entre yemas retenidas y rendimiento no es lineal, lo que indica que un mayor número de yemas retenidas al momento de la poda no se traduce necesariamente en altos rendimientos, debido a que en muchos casos al aumentar la carga de yemas disminuye progresivamente el porcentaje de brotación de las mismas.

Por otra parte, diversos estudios han indicando que la calidad del vino disminuye con suministros hídricos excesivos, ya que aumenta la expresión vegetativa, incrementando el sombreamiento de los racimos, con un consecuente incremento de la acidez total y el pH del mosto, y una reducción significativa del color y de los fenoles totales en el vino (Williams y Matthews, 1990; Goodwin, 1995; Acevedo et al., 2004). En relación a lo anterior, otras investigaciones han indicado que la aplicación de un déficit hídrico controlado puede tener efectos positivos sobre el proceso de maduración de las bayas y calidad del mosto (Koundouras et al., 1997).
Sin embargo, el efecto final del déficit hídrico va a depender de su intensidad y del período fenológico en que se aplique (Goodwin, 1995).

En ensayos realizados para determinar el efecto de distintos niveles de poda y reposición hídrica en el cv. Shiraz, se observó que el rendimiento en las vides regadas se incrementó en forma significativa en la medida que el número de yemas retenidas fue mayor, no existiendo efecto sobre el rendimiento en aquellas plantas sin riego. Por otro lado, el riego incrementó el peso de poda, pero el nivel de poda no tuvo efecto sobre el peso final de sarmientos en las plantas bajo riego (Freeman, 1983). Plantas con mayor expresión vegetativa, debido a un exceso de agua aplicada y a podas con un alto número de yemas, retrasaron la acumulación de azúcar debido a un incremento en el rendimiento; además la acidez y el $\mathrm{pH}$ de las bayas fueron mayores que en aquellas vides sin suplemento hídrico. Esto redujo la proporción de pigmentos totales, afectando el color final del vino. Los resultados de este ensayo son el producto de cinco temporadas consecutivas de evaluación, donde se aplicaron tratamientos extremos de poda (20 y 160 yemas por planta) y suministro hídrico (0 y 60\% de la evaporación de bandeja).

Considerando la escasa información existente sobre el efecto combinado de la poda y el suministro hídrico sobre el desarrollo, calidad y rendimiento del viñedo, la presente investigación se desarrolló con el objetivo de evaluar el efecto combinado de tres niveles de poda y tres niveles de reposición hídrica sobre el crecimiento vegetativo, rendimiento y composición de bayas de un viñedo cv. Cabernet Sauvignon.

\section{MATERIALES Y MÉTODOS}

Se realizó un ensayo en una parcela experimental ubicada en el Valle de Pencahue $\left(35^{\circ} 22^{\prime}\right.$ lat. Sur; $71^{\circ} 47^{\prime}$ long. Oeste; 150 m.s.n.m.), Región del Maule, durante las temporadas vitícolas 2003-2004 y 2004-2005. Se utilizó una parcela de 2,9 ha de cv. Cabernet Sauvignon de 10 años de edad, en un marco de plantación de $3,0 \times 1,2 \mathrm{~m}$, con vides regadas por goteo $\left(3,5 \mathrm{~L} \mathrm{~h}^{-1}\right)$, conducidas en espaldera vertical simple (orientación Norte-Sur), podadas en cordón apitonado y con un peso promedio de poda, previo a la realización de este ensayo, de $800 \mathrm{~g} \mathrm{pl}^{-1}$. 
El suelo presenta una textura franco arenosa y pertenece a la serie Cunculén (Aquic Palexeralfs); la profundidad efectiva de raíces se concentra en los primeros $60 \mathrm{~cm}$. La densidad aparente, capacidad de campo y punto de marchitez permanente son 1,44 $\mathrm{g} \mathrm{cm}^{-3}, 30 \mathrm{~m} \mathrm{~m}^{-3}$ y $11 \mathrm{~m} \mathrm{~m}^{-3}$, respectivamente. El clima es de tipo templado semiárido, caracterizado por un régimen térmico que varía en promedio, entre una máxima de $32,5^{\circ} \mathrm{C}$ y una mínima de $5,5^{\circ} \mathrm{C}$ en el período estival. El régimen hídrico presenta una precipitación promedio anual de $709 \mathrm{~mm}$, un déficit hídrico de $863 \mathrm{~mm}$ con un período seco de siete meses.

Se utilizo un diseño experimental de parcelas subdivididas (strip-plot) con un arreglo factorial de tratamientos ( $3 \times 3)$. Los factores estudiados fueron poda y reposición hídrica con nueve repeticiones. Los niveles de poda fueron 12,18 y 24 yemas por planta y los de reposición hídrica fueron 100,70 y $40 \%$ de la evapotranspiración real del viñedo (ETv). Dichas reposiciones se efectuaron durante el período comprendido entre cuaja y cosecha con una frecuencia de dos riegos semanales, basado en los antecedentes entregados en la publicación de Ortega-Farias et al. (2004a). Cada repetición estuvo constituida por una superficie de $3.249 \mathrm{~m}^{2}$ equivalente a 12 hileras, donde los distintos tratamientos de reposición hídrica se aplicaron a grupos de cuatro hileras, aplicando en éstas los tres niveles de poda señalados.

La ETv se obtuvo diariamente de la siguiente forma:

$\mathrm{ETv}=\mathrm{EB} \mathrm{Kp} \mathrm{Kc}$

donde $\mathrm{EB}=$ bandeja de evaporación $\left(\mathrm{mm} \mathrm{d}^{-1}\right) ; \mathrm{Kp}=$ coeficiente de bandeja $(0,7) ; \mathrm{Kc}=$ coeficiente de cultivo. En este estudio los valores de Kc utilizados para el cv. Cabernet Sauvignon fueron de 0,55 y 0,43 para los períodos fenológicos entre cuajapinta y pinta-cosecha, respectivamente (Ortega-Farias et al., 2004b). Estos valores son utilizados en la zona de Pencahue para obtener vinos de calidad reserva en el cv. Cabernet Sauvignon. De esta forma el tiempo de riego se determinó mediante la siguiente expresión:

$$
\mathrm{TR}=\frac{\mathrm{ETV} A U}{\operatorname{Ne} \mathrm{Ea} q}
$$

donde $\mathrm{TR}=$ tiempo de riego $(\mathrm{h}) ; \mathrm{AU}=$ marco de plantación $\left(\mathrm{m}^{2}\right) ; \mathrm{Ne}=$ número de emisores por plan- ta; Ea = eficiencia de aplicación $(0,9)$ y q = caudal del emisor $\left(\mathrm{L} \mathrm{h}^{-1}\right)$.

Para evaluar el efecto combinado de los distintos niveles de poda y reposición hídrica sobre la expresión vegetativa, en el período de cosecha se midió el largo de sarmientos $(\mathrm{cm})$ y número de nudos por sarmiento, y posterior a la cosecha el peso de poda $(\mathrm{g})$, con lo que se determinó el peso por sarmiento (g). Adicionalmente, con la finalidad de conocer el estado de equilibrio de la vid en cada tratamiento, se realizó el procedimiento descrito por Smart y Robinson (1991), denominado método del cuadrante. Esta técnica consiste en atravesar, la pared vegetativa en forma perpendicular con una varilla metálica de $1 \mathrm{~m}$ de largo y $2 \mathrm{~mm}$ de diámetro, con la ayuda de una regleta de PVC perforada cada $10 \mathrm{~cm}$, dispuesta en forma horizontal, paralela a la pared del follaje a la altura de los racimos. Con esta metodología, inmediatamente previo a la cosecha, se estimó el número de capas de hojas $(\mathrm{NCH})$ y el porcentaje de racimos expuestos, los cuales se utilizaron para caracterizar el vigor de las distintas unidades experimentales considerando un total de 40 inserciones por cada unidad experimental.

Al momento de la cosecha se midieron los componentes del rendimiento tales como la producción por planta $(\mathrm{kg})$, número de racimos por planta ( $\sin$ considerar pámpanos), número de racimos por brote, peso de racimos (g) y número de bayas por racimo. Con una muestra representativa de 200 bayas por cada repetición en cada tratamiento, se midió la composición de las bayas en base a la determinación de sólidos solubles (a través de refractometría), acidez total (neutralización con $\mathrm{NaOH} 0,1 \mathrm{~N}$, expresado en $\mathrm{g} \mathrm{L}^{-1}$ de ácido sulfúrico) y $\mathrm{pH}$ (mediante potenciometría). Además, se determinó el diámetro de bayas $(\mathrm{mm})$, la relación cutícula/pulpa y la concentración de antocianas y polifenoles totales $\left(\mathrm{mg} \mathrm{L}^{-1}\right)$ en las bayas a través del método de Glories (Marquette, 1999). Posteriormente, y una vez realizada la poda invernal, se determinó el índice de Ravaz (kg de fruta/kg de poda) para cada uno de los tratamientos.

Los resultados obtenidos se sometieron a un análisis de varianza (ANDEVA), y en los casos en donde existieron diferencias significativas entre los tratamientos se realizó la prueba de comparación de medias de Duncan con un intervalo de confianza de $95 \%$. 


\section{RESULTADOS Y DISCUSIÓN}

Desde brotación a cosecha en la primera temporada precipitaron $120,3 \mathrm{~mm}$, de los cuales el 99,4; 0,4 y $0,2 \%$ se concentraron en los períodos de brotación-cuaja, cuaja-pinta y pinta-cosecha, respec- tivamente (Figura 1). Por el contrario, en la segunda temporada, con un total de $131,4 \mathrm{~mm}$, las precipitaciones se distribuyeron en forma más uniforme, con $61,5 \%$ del agua caída en el período de brotación-cuaja, un $20,9 \%$ en el período de cuaja-pinta, y un $17,6 \%$ en el período de pinta-cosecha. En

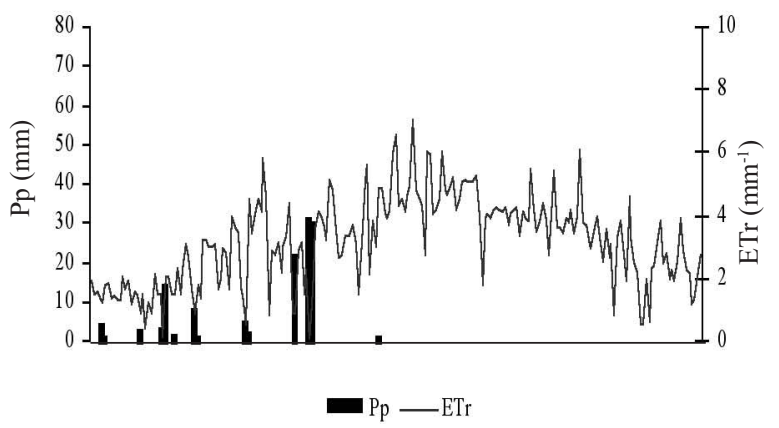

2003-2004

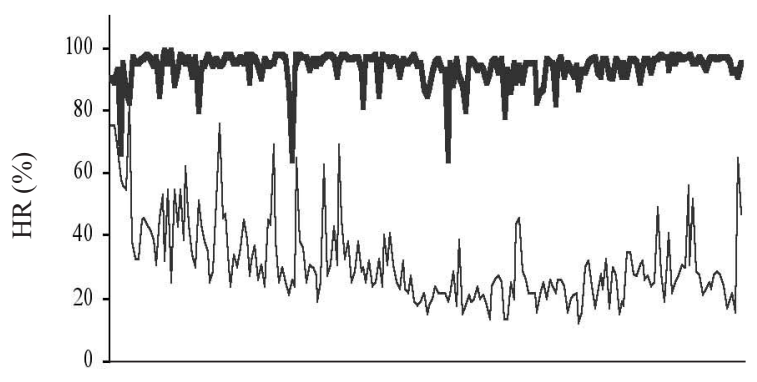

-HRmáx —HRmín

2003-2004

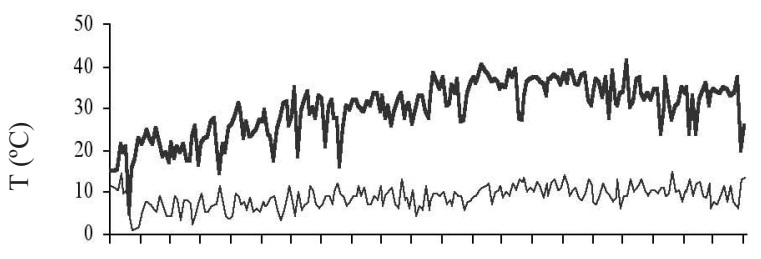

1- 13- 25- 7- 19-31- 12- 24- 6- 18- 30- 11- 23- 4- 16- 28- 11- 23sep sep sep oct oct oct nov nov dic dic dic ene ene feb feb feb mar mar Fecha

$$
\text { —Tmáx — Tmin }
$$

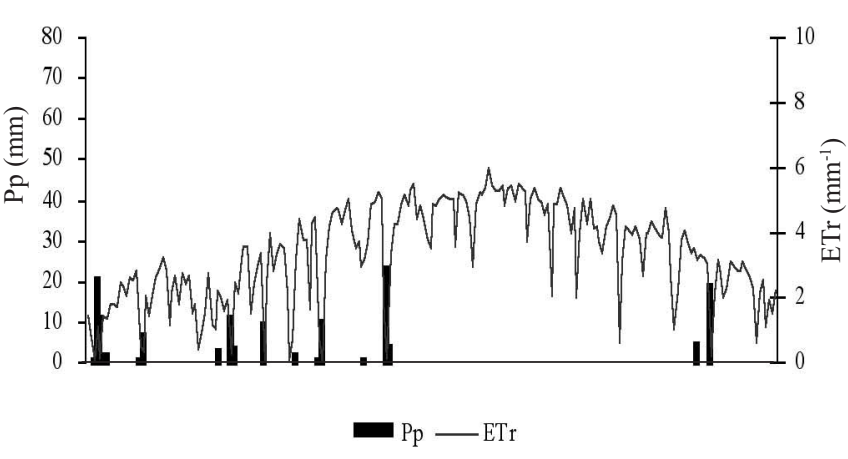

2004-2005

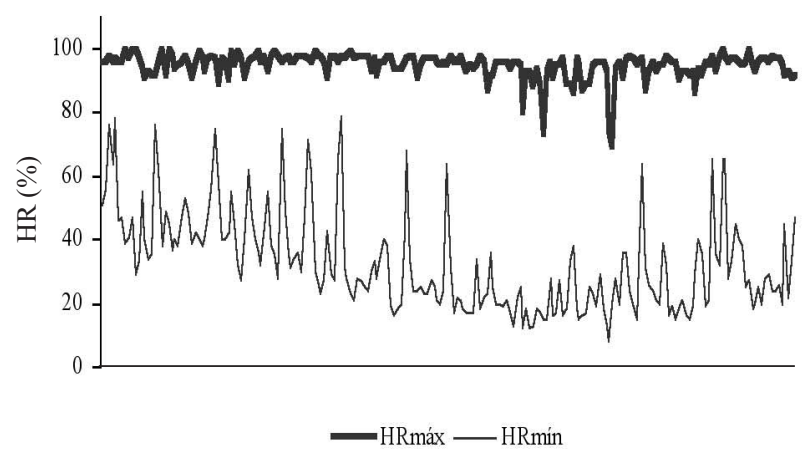

2004-2005

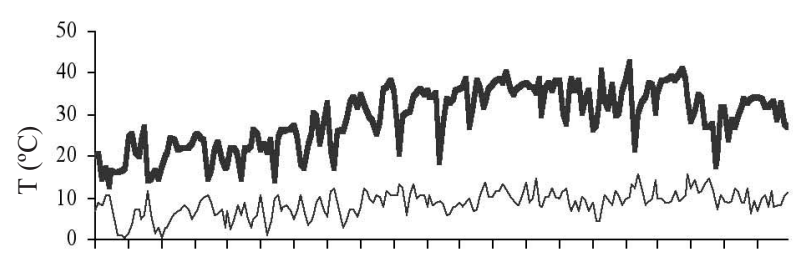

1- 13- 25- 7- 19-31- 12- 24- 6- 18- 30- 11- 23- 4- 16- 28- 12- 24sep sep sep oct oct oct nov nov dic dic dic ene ene feb feb feb mar mar Fecha

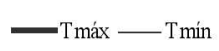

Figura 1. Precipitación (Pp), evapotranspiración de referencia $(\mathrm{ETr})$, temperatura (máxima $=$ Tmax y mínima $=$

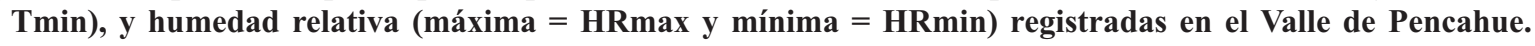
Temporadas 2003-2004 y 2004-2005.

Figure 1. Precipitation (Pp), reference evapotranspiration (ETr), temperature (maximum $=$ Tmax and minimum $=\mathbf{T m i n}$ ), and relative humidity (maximum $=H R \max$ and $\operatorname{minimum}=H R \min$ ) recorded at Pencahue Valley. 2003-2004 and 2004-2005 growing seasons. 
ambas temporadas se observó una mayor demanda hídrica en los meses de noviembre a febrero, con valores acumulados desde brotación a cosecha de evapotranspiración de referencia $(\mathrm{ETr}=\mathrm{EB} \mathrm{Kp})$ de 670 y $704 \mathrm{~mm}$ para el primer y segundo año de evaluación, respectivamente.

En relación a la temperatura y humedad relativa registradas durante las temporadas 2003-2004 y 2004-2005, se observaron temperaturas mínimas en septiembre de 0,8 y $0,4^{\circ} \mathrm{C}$ y valores máximos en febrero de 41,2 y $42,7^{\circ} \mathrm{C}$, en ambas temporadas de estudio. La situación anterior contrasta con los valores de humedad relativa registrados, en donde los valores menores fueron observados en febrero (12 y $8 \%$ ) para ambas temporadas. Por último, es importante señalar que se observó una relación directa entre los valores máximos de temperatura y los valores máximos de ETr en ambas temporadas de riego, siendo los meses de diciembre a febrero los de mayor demanda hídrica.

Los volúmenes totales de agua aplicada desde brotación a cosecha durante todo el período de riego en el tratamiento T1 (100\% ETv), en las temporadas 2003-2004 y 2004-2005, fueron de 1.831 y $2.042 \mathrm{~m}^{3} \mathrm{ha}^{-1}$, respectivamente (Figura 2). Acevedo et al. (2004) aplicaron volúmenes similares en
Cabernet Sauvignon en la zona de Pencahue con un clima templado semiárido. Por otra parte, Ferreyra et al. (1998) y Gurovich (1998) aplicaron 4.480 y $4.762 \mathrm{~m}^{3} \mathrm{ha}^{-1}$, respectivamente, en Cabernet Sauvignon producido en condiciones semiáridas en la zona de Pirque y la Pintana, para el tratamiento $100 \%$ ETv, respectivamente.

En cuanto al crecimiento vegetativo (Cuadro 1), el análisis estadístico indicó que sólo existieron diferencias altamente significativas $(p \leq 0,01)$ en el peso individual del sarmiento, y significativas $(\mathrm{p} \leq 0,05)$ en el largo del sarmiento, entre los tratamientos con distinta poda durante la primera temporada, observándose que aquellas plantas en las que se dejó un menor número de yemas, presentaron un mayor peso y largo de sarmiento, lo cual concuerda con los resultados obtenidos por Miller et al. (1996) y Smithyman et al. (2001), quienes observaron la misma tendencia. Esto se debe a que al limitar los puntos de crecimiento, las reservas de carbohidratos y las sustancias aportadas por las raíces son adecuadas para soportar la máxima tasa de crecimiento de los brotes. Por otro lado, en la medida que el número de puntos de crecimiento se incrementa, comienzan a competir entre ellos por los carbohidratos disponibles, agua, nutrientes y citoquininas (Miller et al., 1996).

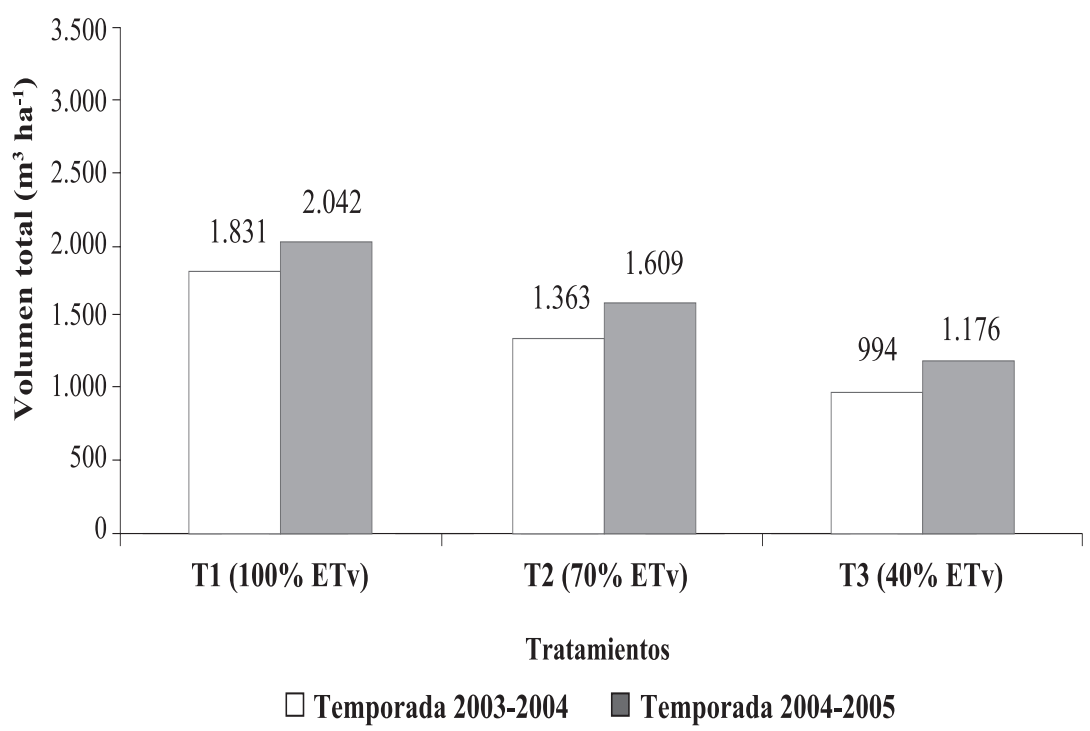

Figura 2. Volumen total de agua aplicada $\left(\mathrm{m}^{3} \mathrm{ha}^{-1}\right)$ para los distintos tratamientos de reposición hídrica en vides $\mathrm{cv}$. Cabernet Sauvignon en el Valle de Pencahue. Temporadas 2003-2004 y 2004-2005. ETv: evapotranspiración real de la viña.

Figure 2. Total volume of water applied $\left(\mathrm{m}^{3} \mathrm{ha}^{-1}\right)$ for the different treatments of water application in grapevines $\mathrm{cv}$. Cabernet Sauvignon in the Pencahue Valley. 2003-2004 and 2004-2005 growing seasons. ETv: real evapotranspiration of vineyard. 
Cuadro 1. Efecto de distintos niveles de poda y reposición hídrica sobre el crecimiento vegetativo en vides cv. Cabernet Sauvignon, Valle de Pencahue. Temporadas 2003-2004 y 2004-2005.

Table 1. Effect of different pruning levels and water application on vegetative growth in Cabernet Sauvignon grapevines, Pencahue Valley. 2003-2004 and 2004-2005 growing seasons.

\begin{tabular}{|c|c|c|c|c|}
\hline Tratamiento & $\begin{array}{l}\text { Largo sarmiento } \\
(\mathbf{c m})\end{array}$ & $N^{\circ}$ nudos & $\begin{array}{l}\text { Peso de poda } \\
\quad\left(\mathrm{g} \mathrm{pl}^{-1}\right)\end{array}$ & $\begin{array}{l}\text { Peso por } \\
\text { sarmiento } \\
\quad(g)\end{array}$ \\
\hline \multicolumn{5}{|l|}{$2003-2004$} \\
\hline \multicolumn{5}{|l|}{ Poda, yemas $\mathrm{pl}^{-1}$} \\
\hline 24 & $141,7 \mathrm{a}$ & 22,6 & 802,0 & $33,4 \mathrm{a}$ \\
\hline 18 & $147,4 a b$ & 23,0 & 717,5 & $39,9 \mathrm{~b}$ \\
\hline 12 & $154,8 \mathrm{~b}$ & 28,8 & 732,8 & $47,7 \mathrm{c}$ \\
\hline Significancia & $*$ & n.s. & n.s. & $* *$ \\
\hline \multicolumn{5}{|l|}{ Reposición hídrica } \\
\hline $100 \% \mathrm{ETv}$ & 152,7 & 27,6 & 828,4 & 43,0 \\
\hline $70 \% \mathrm{ETv}$ & 147,4 & 23,2 & 685,5 & 38,8 \\
\hline $40 \% \mathrm{ETV}$ & 143,7 & 23,2 & 738,3 & 39,2 \\
\hline Significancia & n.s. & n.s. & n.s. & n.s. \\
\hline Interacción A x B & n.s. & n.s. & n.s. & n.s. \\
\hline \multicolumn{5}{|l|}{$2004-2005$} \\
\hline \multicolumn{5}{|l|}{ Poda, yemas $\mathrm{pl}^{-1}$} \\
\hline 24 & 147,1 & 23,3 & $1.031,6 \mathrm{a}$ & 43,0 \\
\hline 18 & 149,4 & 23,0 & $612,9 \mathrm{~b}$ & 34,1 \\
\hline 12 & 154,2 & 23,5 & $523,4 \mathrm{~b}$ & 43,6 \\
\hline Significancia & n.s. & n.s. & $* *$ & n.s. \\
\hline \multicolumn{5}{|l|}{ Reposición hídrica } \\
\hline $100 \% \mathrm{ETV}$ & 160,4 & 24,3 & 734,2 & 41,4 \\
\hline $70 \% \mathrm{ETV}$ & 145,9 & 22,8 & 699,5 & 39,1 \\
\hline $40 \% \mathrm{ETv}$ & 144,4 & 22,8 & 734,2 & 40,2 \\
\hline Significancia & n.s. & n.s. & n.s. & n.s. \\
\hline Interacción A x B & n.s. & n.s. & n.s. & n.s. \\
\hline
\end{tabular}

Valores con distinta letra en las columnas indican que hubo diferencias significativas de acuerdo a la prueba de comparación múltiple de Duncan ( $\mathrm{p} \leq 0,05)$.

n.s.: no significativo; $*$ : significativo $(\mathrm{p} \leq 0,05)$; $* *$ : altamente significativo $(\mathrm{p} \leq 0,01)$.

ETv: evapotranspiración real del viñedo.

No se encontraron diferencias significativas en peso de poda entre los distintos tratamientos de poda durante la primera temporada de evaluación. Freeman (1983) tampoco detectó diferencias entre los distintos niveles de poda en temporadas analizadas individualmente, y señaló que un cambio en la severidad de la poda afectaría más bien al peso promedio individual de los brotes y no necesariamente al peso total de poda por planta, tal como ocurrió durante la primera temporada de evaluación en este ensayo. No obstante, en el análisis combinado de los 5 años de datos del caso anterior, el peso de poda fue significativamente afectado por el nivel de poda, lo cual puede explicar los resultados obtenidos en la segunda temporada de esta investiga- ción, en la cual se registraron diferencias altamente significativas, donde se obtuvo un mayor peso de poda a medida que fue mayor el número de yemas retenidas.

En relación a los tratamientos de reposición hídrica, no se encontraron diferencias significativas para el crecimiento vegetativo en este cultivar, a pesar de la aplicación de los distintos niveles de agua aplicada durante las dos temporadas de evaluación. Estos resultados discrepan de los obtenidos por Dry y Loveys (1999), quienes encontraron diferencias significativas en las variables de largo de brotes, largo de entrenudos y peso de poda para las aplicaciones de $100 \%$ ETv y $0 \%$ ETv durante el período 
Cuadro 2. Efecto de distintos niveles de poda y reposición hídrica sobre la caracterización de la pared del follaje en vides cv. Cabernet Sauvignon, Valle de Pencahue. Temporadas 2003-2004 y 2004-2005.

Table 2. Effect of different pruning levels and water application on characterization of canopy in Cabernet Sauvignon grapevines, Pencahue Valley. 2003-2004 and 2004-2005 growing seasons.

\begin{tabular}{|c|c|c|c|c|c|c|}
\hline \multirow[t]{3}{*}{ Tratamiento } & \multicolumn{6}{|c|}{ Temporada } \\
\hline & \multicolumn{3}{|c|}{ 2003-2004 } & \multicolumn{3}{|c|}{ 2004-2005 } \\
\hline & $\begin{array}{l}N^{\circ} \text { capas } \\
\text { de hojas }\end{array}$ & $\begin{array}{c}\text { Espacios } \\
(\%)\end{array}$ & $\begin{array}{c}\text { Racimos } \\
\text { expuestos } \\
(\%)\end{array}$ & $\begin{array}{l}N^{\circ} \text { capas } \\
\text { de hojas }\end{array}$ & $\begin{array}{c}\text { Espacios } \\
(\%)\end{array}$ & $\begin{array}{c}\text { Racimos } \\
\text { expuestos } \\
(\%)\end{array}$ \\
\hline \multicolumn{7}{|l|}{ Poda, yemas $\mathrm{pl}^{-1}$} \\
\hline 24 & $2,1 \mathrm{a}$ & $2,3 \mathrm{a}$ & 39,9 a & $2,2 \mathrm{a}$ & 3,6 a & $62,2 \mathrm{~b}$ \\
\hline 18 & $1,7 \mathrm{~b}$ & $5,4 \mathrm{~b}$ & $65,2 \mathrm{~b}$ & $1,4 \mathrm{~b}$ & $17,0 \mathrm{~b}$ & $67,1 \mathrm{~b}$ \\
\hline 12 & $1,4 \mathrm{c}$ & $12,6 \mathrm{c}$ & $87,7 \mathrm{c}$ & $1,2 \mathrm{~b}$ & $19,0 \mathrm{~b}$ & $87,1 \mathrm{a}$ \\
\hline Significancia & $* *$ & $* *$ & $* *$ & $* *$ & $* *$ & $* *$ \\
\hline \multicolumn{7}{|l|}{ Reposición hídrica } \\
\hline $100 \%$ ETv & $1,9 \mathrm{a}$ & $4,3 \mathrm{a}$ & 58,8 & $2,1 \mathrm{a}$ & $8,7 \mathrm{a}$ & $68,8 \mathrm{a}$ \\
\hline $70 \% \mathrm{ETV}$ & $1,9 \mathrm{a}$ & $5,7 \mathrm{a}$ & 66,5 & $1,6 \mathrm{~b}$ & $15,5 \mathrm{~b}$ & $73,9 \mathrm{ab}$ \\
\hline $40 \% \mathrm{ETV}$ & $1,5 \mathrm{~b}$ & $10,3 \mathrm{~b}$ & 64,5 & $1,3 \mathrm{~b}$ & $15,4 \mathrm{~b}$ & $83,6 \mathrm{~b}$ \\
\hline Significancia & $* *$ & $* *$ & n.s. & $* *$ & $*$ & $*$ \\
\hline Interacción A x B & $*$ & n.s. & n.s. & n.s. & n.s. & n.s. \\
\hline
\end{tabular}

Valores con distinta letra en las columnas indican que hubo diferencias significativas de acuerdo a la prueba de comparación múltiple de Duncan $(\mathrm{p} \leq 0,05)$.

ETv: evapotranspiración real del viñedo; n.s.: no significativo; *: significativo $(\mathrm{p} \leq 0,05)$; **: altamente significativo $(\mathrm{p} \leq 0,01)$.

comprendido entre cuaja y cosecha. Esto se debió a que los mayores tiempos de riego en este ensayo fueron aplicados en el período de pinta, momento en que los requerimientos hídricos de la vid son mayores; a su vez, en este momento se detiene el crecimiento de los brotes y comienza el proceso de lignificación de éstos (Smart y Coombe, 1983; Williams y Mathews, 1990), coincidiendo con los resultados obtenidos por Poni et al. (1994) y Ginestar et al. (1998a), quienes indicaron que la aplicación de déficit hídrico entre pinta y cosecha no tendría efectos significativos sobre el crecimiento de brotes.

Con respecto al número de capas de hojas, porcentaje de espacios y de racimos expuestos (Cuadro 2), se observó para las dos temporadas diferencias altamente significativas ( $\mathrm{p} \leq 0,01)$ entre los tratamientos de poda en las tres variables anteriormente mencionadas, encontrándose un mayor número de capas de hojas, menor porcentaje de espacios y de racimos expuestos en aquellas plantas donde se dejó un mayor número de yemas al momento de la poda, en comparación con aquellas donde se retuvo un menor número. Por otra parte, en los tratamientos de reposición hídrica se encontraron diferencias altamente significativas durante ambas tem- poradas para el número de capas de hojas y el porcentaje de espacios durante la primera, y significativas $(\mathrm{p} \leq 0,05)$ durante la segunda temporada. Finalmente, en el porcentaje de racimos expuestos se observaron diferencias significativas sólo en la segunda temporada, cuando las vides sometidas a un mayor déficit hídrico presentaron un menor desarrollo de follaje con la consecuente disminución en el número de capas de hojas, con un mayor porcentaje de espacios y de racimos expuestos. Además, en la primera temporada se presentó una interacción positiva para el número de capas de hojas (Figura 3), en la cual con una poda más severa y menores reposiciones hídricas se generó un menor número de capas de hojas. Ginestar et al. (1998a) también encontraron diferencias significativas entre los tratamientos de reposición hídrica (0 y 50\% ETv), observando un mayor porcentaje de racimos expuestos, en cosecha, para vides sin aplicación de agua de riego.

En cuanto a los componentes de rendimiento, se observaron diferencias altamente significativas $(\mathrm{p} \leq 0,01)$ en rendimiento y número de racimos por planta entre los distintos tratamientos de poda durante ambas temporadas, y diferencias altamente significativas en la primera temporada para el ín- 


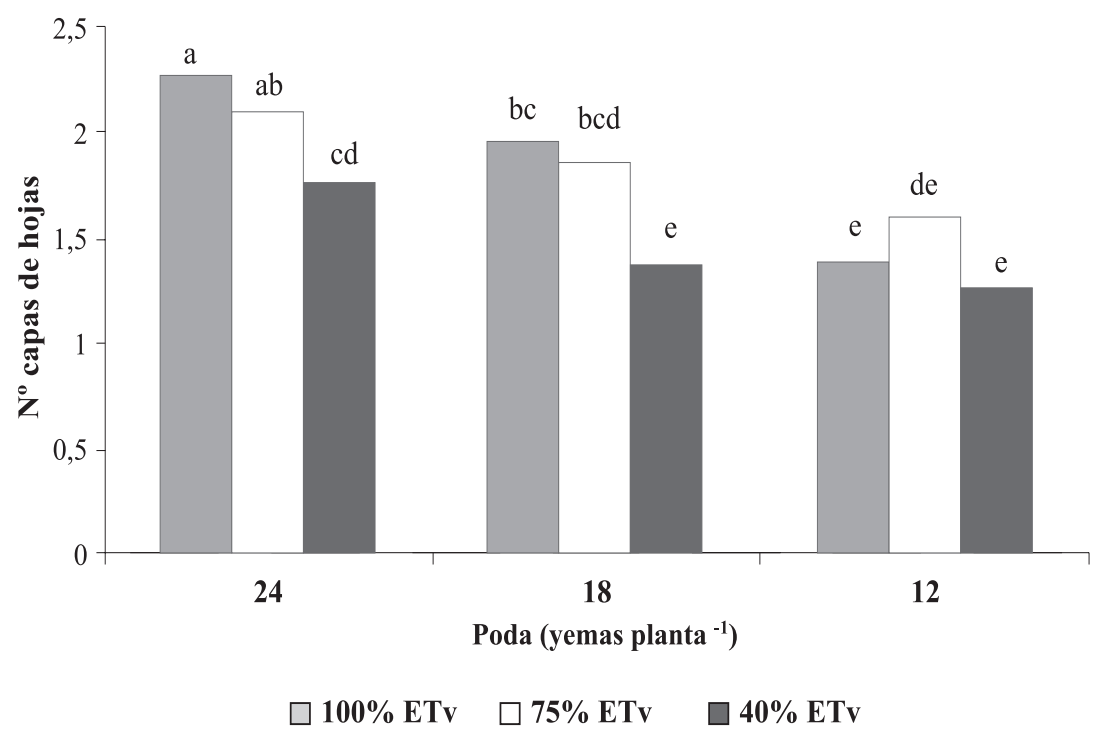

Figura 3. Efecto de los tratamientos con distintos niveles de poda y reposición hídrica sobre el número de capas de hojas en vides cv. Cabernet Sauvignon en el Valle de Pencahue. Temporada 2003-2004. ETv: evapotranspiración real de la viña.

Figure 3. Effect of different pruning levels and water application on the leaf layer number for grapevines cv. Cabernet Sauvignon in the Pencahue Valley. 2003-2004 growing season.

* Letras iguales indica que no existen diferencias significativas entre tratamientos.

ETv: real evapotranspiration of vineyard.

dice de Ravaz (IR) y peso de racimo, y para el número de racimos por brote en la segunda temporada (Cuadro 3). Al respecto se puede observar que las vides en las cuales se dejó un mayor número de yemas, presentaron un mayor número de racimos por planta, de menor peso, con un mayor rendimiento final en comparación a aquellas en que se retuvo un menor número de yemas. Esto último estaría explicando el mayor IR observado en las vides con un mayor número de yemas durante la primera temporada, debido a que en ésta los valores obtenidos en peso de poda para los distintos tratamientos no difirieron estadísticamente (Cuadro 1). Lo anterior no ocurrió en la segunda temporada, donde una disminución en rendimiento de la fruta por planta fue acompañada de una disminución importante en el peso de poda en los tratamientos evaluados, lo que no permitió encontrar diferencias en el IR. De este modo, es importante señalar que resultados obtenidos por Bravdo et al. (1985) indicaron que la mejor calidad de vino fue obtenida con valores de IR que variaron entre 3 y 10 . Por el contrario, índices mayores a 10 resultaron en una situación de sobreproducción, en la cual las vides no fueron capaces de sustentar una adecuada madurez de la fruta.
En relación a la reposición hídrica, existieron diferencias significativas en ambas temporadas para el peso de racimos, diferencias altamente significativas en rendimiento y número de racimos por planta, y significativa para el número de racimos por brote durante la segunda temporada en los tratamientos con mayor reposición hídrica. Similares resultados fueron encontrados por Goodwin (1995), Nadal y Arola (1995) y Ferreyra et al. (1998), quienes observaron que el rendimiento disminuyó significativamente para el tratamiento sin riego en comparación con el 100\% ETv. De la misma manera, y basados en resultados similares, Kliewer et al. (1983) señalan que el riego aumenta tanto el número de bayas por racimo como el de racimos por planta.

La mayor cantidad de variables con diferencias significativas encontradas entre los tratamientos de riego aplicados durante la segunda temporada, pudieron deberse a que el estrés hídrico promovería una mayor fertilidad de yemas, al producirse una mayor inducción de las mismas durante la temporada anterior, debido al menor sombreamiento en las plantas, provocado por un menor follaje (Kliewer et al. 1983). 
Cuadro 3. Efecto de distintos niveles de poda y reposición hídrica sobre el rendimiento y sus componentes en vides cv. Cabernet Sauvignon, Valle de Pencahue. Temporadas 2003-2004 y 2004-2005.

Table 3. Effect of different pruning levels and water application on yield and its components in Cabernet Sauvignon grapevines, Pencahue Valley. 2003-2004 and 2004-2005 growing seasons.

\begin{tabular}{|c|c|c|c|c|c|c|}
\hline Tratamiento & $\begin{array}{c}\mathrm{N}^{\circ} \\
\text { racimos }\end{array}$ & $\begin{array}{l}\text { Racimos } \\
\text { por brote }\end{array}$ & $\begin{array}{c}\text { Peso racimos } \\
(\mathrm{kg})\end{array}$ & $\begin{array}{c}\mathrm{N}^{\circ} \\
\text { bayas }\end{array}$ & $\begin{array}{c}\text { Rendimiento } \\
\left(\mathrm{kg} \mathrm{pl}^{-1}\right)\end{array}$ & $\begin{array}{l}\text { Índice de } \\
\text { Ravaz }\end{array}$ \\
\hline \multicolumn{7}{|l|}{ 2003-2004 } \\
\hline \multicolumn{7}{|l|}{ Poda, yemas $\mathrm{pl}^{-1}$} \\
\hline 24 & $35,3 \mathrm{a}$ & 1,5 & $0,11 \mathrm{a}$ & 117,3 & $3,4 \mathrm{a}$ & $4,3 \mathrm{a}$ \\
\hline 18 & $23,9 \mathrm{~b}$ & 1,3 & $0,12 \mathrm{~b}$ & 115,9 & $2,4 \mathrm{~b}$ & $3,5 \mathrm{~b}$ \\
\hline 12 & $16,9 \mathrm{c}$ & 1,4 & $0,14 \mathrm{~b}$ & 111,6 & $1,6 \mathrm{c}$ & $2,9 \mathrm{~b}$ \\
\hline Significancia & $* *$ & n.s. & $* *$ & n.s. & $* *$ & $* *$ \\
\hline \multicolumn{7}{|l|}{ Reposición hídrica } \\
\hline $100 \%$ ETv & 25,1 & 1,4 & $0,13 \mathrm{a}$ & 118,0 & 2,6 & 3,5 \\
\hline $70 \% \mathrm{ETV}$ & 26,7 & 1,5 & $0,12 \mathrm{ab}$ & 113,2 & 2,5 & 3,6 \\
\hline $40 \%$ ETv & 24,3 & 1,3 & $0,11 \mathrm{~b}$ & 113,6 & 2,3 & 3,5 \\
\hline Significancia & n.s. & n.s. & $*$ & n.s. & n.s. & n.s. \\
\hline Interacción A x B & n.s. & n.s. & n.s. & n.s. & n.s. & n.s. \\
\hline \multicolumn{7}{|l|}{$2004-2005$} \\
\hline \multicolumn{7}{|l|}{ Poda, yemas $\mathrm{pl}^{-1}$} \\
\hline 24 & $42,9 \mathrm{a}$ & $1,8 \mathrm{~b}$ & 0,07 & 72,1 & $3,2 \mathrm{a}$ & 2,9 \\
\hline 18 & $24,2 \mathrm{~b}$ & $1,4 \mathrm{a}$ & 0,07 & 68,8 & $1,7 \mathrm{~b}$ & 3,0 \\
\hline 12 & $23,4 \mathrm{~b}$ & $2,0 \mathrm{~b}$ & 0,07 & 79,4 & $1,9 \mathrm{~b}$ & 3,2 \\
\hline Significancia & $* *$ & $* *$ & n.s. & n.s. & $* *$ & n.s. \\
\hline \multicolumn{7}{|l|}{ Reposición hídrica } \\
\hline $100 \%$ ETv & 34,6 a & $2,0 \mathrm{a}$ & $0,08 \mathrm{a}$ & 75,7 & $3,0 \mathrm{a}$ & 3,6 \\
\hline $70 \% \mathrm{ETv}$ & $29,1 \mathrm{~b}$ & $1,6 \mathrm{~b}$ & $0,06 \mathrm{~b}$ & 73,0 & $2,0 \mathrm{~b}$ & 2,8 \\
\hline $40 \%$ ETv & $26,9 \mathrm{~b}$ & $1,6 \mathrm{~b}$ & $0,06 \mathrm{~b}$ & 71,6 & $1,8 \mathrm{~b}$ & 2,6 \\
\hline Significancia & $* *$ & $*$ & $*$ & n.s. & $* *$ & n.s. \\
\hline Interacción A x B & n.s. & n.s. & n.s. & n.s. & n.s. & n.s \\
\hline
\end{tabular}

Valores con distinta letra en las columnas indican que no hubo diferencias significativas de acuerdo a la prueba de comparación múltiple de Duncan $(\mathrm{p} \leq 0,05)$.

ETv: evapotranspiración real del viñedo; n.s.: no significativo; *: significativo $(\mathrm{p} \leq 0,05)$; **: altamente significativo (p $\leq 0,01)$.

El análisis estadístico indicó que en ambas temporadas no existieron diferencias significativas entre los tratamientos de reposición hídrica y poda para la acidez total, pH y sólidos solubles (Cuadro 4). Estos últimos resultados coinciden con los obtenidos por Freeman (1983), quien señaló que los distintos tratamientos de poda no tuvieron efecto sobre la acidez titulable y madurez a cosecha, durante cinco temporadas consecutivas de evaluaciones. Ortega-Farias et al. (2004a) no encontraron un efecto significativo del estrés hídrico sobre los sólidos solubles y pH. Por otro lado, Hepner et al. (1985), Naor et al. (1993), Koundouras et al. (1997) y Sipiora y Gutierrez (1998) observaron una mayor concentración de sólidos solubles ( ${ }^{\circ}$ Brix) en tratamientos con menor reposición hídrica.
Con respecto a las variables de composición de bayas a la cosecha (Cuadro 5), no se observaron diferencias significativas entre los distintos niveles de poda ni de reposición hídrica durante la primera temporada de evaluación. Sin embargo, en la segunda temporada existieron diferencias significativas entre los tratamientos de poda para las antocianas totales, observándose racimos con un mayor contenido de éstas en aquellos tratamientos con podas más severas. Este incremento en la concentración de antocianas se puede deber a la mayor exposición de racimos a la radiación directa, debido a una menor expresión vegetativa (Ginestar et al., 1998b; Acevedo et al., 2004), como se observa en el Cuadro 2, en donde tratamientos con un menor número de capas de hojas y un mayor porcen- 
Cuadro 4. Efecto de distintos niveles de poda y reposición hídrica sobre los componentes de madurez de las bayas en vides cv. Cabernet Sauvignon, Valle de Pencahue. Temporadas 2003-2004 y 2004-2005.

Table 4. Effect of different pruning levels and water application on grape maturity components in Cabernet Sauvignon grapevines, Pencahue Valley. 2003-2004 and 2004-2005 growing seasons.

\begin{tabular}{|c|c|c|c|}
\hline Tratamiento & Acidez total & pH & Sólidos solubles $\left({ }^{\circ} \mathrm{Brix}\right)$ \\
\hline \multicolumn{4}{|l|}{$2003-2004$} \\
\hline \multicolumn{4}{|l|}{ Poda, yemas $\mathrm{pl}^{-1}$} \\
\hline 24 & 3,2 & 3,6 & 24,6 \\
\hline 18 & 3,1 & 3,6 & 24,5 \\
\hline 12 & 3,0 & 3,6 & 24,8 \\
\hline Significancia & $*$ & n.s. & n.s. \\
\hline \multicolumn{4}{|l|}{ Reposición hídrica } \\
\hline $100 \%$ ETv & 3,1 & 3,6 & 24,2 \\
\hline $70 \% \mathrm{ETv}$ & 3,0 & 3,6 & 24,8 \\
\hline $40 \%$ ETv & 3,1 & 3,6 & 25,0 \\
\hline Significancia & n.s. & n.s. & n.s. \\
\hline Interacción A x B & n.s. & n.s. & n.s. \\
\hline \multicolumn{4}{|l|}{$2004-2005$} \\
\hline \multicolumn{4}{|l|}{ Poda, yemas $\mathrm{pl}^{-1}$} \\
\hline 24 & 3,5 & 3,6 & 23,4 \\
\hline 18 & 3,4 & 3,6 & 23,8 \\
\hline 12 & 3,3 & 3,6 & 24,0 \\
\hline Significancia & n.s. & n.s. & n.s \\
\hline \multicolumn{4}{|l|}{ Reposición hídrica } \\
\hline $100 \%$ ETv & 3,5 & 3,6 & 23,4 \\
\hline $70 \% \mathrm{ETv}$ & 3,5 & 3,6 & 23,9 \\
\hline $40 \% \mathrm{ETv}$ & 3,3 & 3,6 & 24,0 \\
\hline Significancia & n.s. & n.s. & n.s. \\
\hline Interacción A x B & n.s. & n.s. & n.s. \\
\hline
\end{tabular}

ETv: evapotranspiración real del viñedo; n.s.: no significativo; *: significativo ( $\leq \leq 0,05)$; **: altamente significativo $(\mathrm{p} \leq 0,01)$.

taje de racimos expuestos presentan la mayor concentración de antocianas totales, debido a un efecto directo de la radiación solar en la composición de las bayas, a través del aumento en la síntesis de pigmentos antociánicos debido al aumento de la temperatura en las bayas (Bergqvist et al., 2001). No obstante lo anterior, es posible que los rangos de intensidad de poda estudiados no fueran lo suficientemente amplios como para establecer diferencias significativas en la composición de bayas, pues los tratamientos fueron adaptados al criterio productivo y comercial de la viña.

En cuanto al riego se encontraron diferencias significativas para el diámetro de baya, y diferencias altamente significativas para las antocianas totales, donde vides sometidas a una menor reposición hídrica presentaron bayas más pequeñas con una mayor concentración de antocianas totales. De este modo, el mayor contenido de antocianas se relaciona directamente con una mayor relación cutícula/pulpa de las bayas; tal como lo señalan Tregoat et al. (2002), quienes encontraron mayor concentración de polifenoles en bayas de menor peso y diámetro, aumentando de esta forma su relación cutícula/pulpa. Por otro lado, los resultados en los compuestos fenólicos en las bayas son similares a los obtenidos por Koundouras et al. (1997), Ginestar et al. (1998b), Choné et al. (2001), y Kennedy et al. (2002), quienes observaron una mayor concentración de fenoles y antocianas en bayas de plantas sometidas a déficit hídrico controlado. 
Cuadro 5. Efecto de distintos niveles de poda y reposición hídrica sobre la composición de las bayas en vides cv. Cabernet Sauvignon, Valle de Pencahue. Temporadas 2003-2004 y 2004-2005.

Table 5. Effect of different pruning levels and water application on grape composition in Cabernet Sauvignon grapevines, Pencahue Valley. 2003-2004 and 2004-2005 growing seasons.

\begin{tabular}{|c|c|c|c|c|}
\hline Tratamiento & $\begin{array}{l}\text { Diámetro de bayas } \\
\qquad(\mathrm{mm})\end{array}$ & $\begin{array}{c}\text { Relación } \\
\text { cutícula/pulpa }\end{array}$ & $\begin{array}{l}\text { Antocianas totales } \\
\qquad\left(\mathrm{mg} \mathrm{L}^{-1}\right)\end{array}$ & $\begin{array}{l}\text { Polifenoles totales } \\
\qquad\left(\mathrm{mg} \mathrm{L}^{-1}\right)\end{array}$ \\
\hline \multicolumn{5}{|l|}{ 2003-2004 } \\
\hline \multicolumn{5}{|l|}{ Poda, yemas $\mathrm{pl}^{-1}$} \\
\hline 24 & 11,1 & 4,3 & 1.507 & 2.497 \\
\hline 18 & 11,3 & 4,2 & 1.481 & 2.423 \\
\hline 12 & 10,9 & 4,1 & 1.543 & 2.596 \\
\hline Significancia & n.s. & n.s. & n.s. & n.s. \\
\hline \multicolumn{5}{|l|}{ Reposición hídrica } \\
\hline $100 \%$ ETv & 11,2 & 4,2 & 1.487 & 2.440 \\
\hline $70 \% \mathrm{ETv}$ & 11,2 & 4,1 & 1.496 & 2.466 \\
\hline $40 \% \mathrm{ETv}$ & 10,9 & 4,3 & 1.547 & 2.609 \\
\hline Significancia & n.s. & n.s. & n.s. & n.s. \\
\hline Interacción A x B & n.s. & n.s. & n.s. & n.s. \\
\hline \multicolumn{5}{|l|}{$2004-2005$} \\
\hline \multicolumn{5}{|l|}{ Poda, yemas $\mathrm{pl}^{-1}$} \\
\hline 24 & 10,1 & 4,6 & $1.364 \mathrm{~b}$ & 2.377 \\
\hline 18 & 10,1 & 4,7 & $1.354 \mathrm{~b}$ & 2.457 \\
\hline 12 & 10,0 & 4,9 & $1.506 \mathrm{a}$ & 2.338 \\
\hline Significancia & n.s. & n.s. & $*$ & n.s. \\
\hline \multicolumn{5}{|l|}{ Reposición hídrica } \\
\hline $100 \%$ ETv & $10,4 \mathrm{a}$ & 4,8 & $1.294 \mathrm{a}$ & 2.340 \\
\hline $70 \% \mathrm{ETV}$ & $9,9 \mathrm{~b}$ & 4,6 & $1.411 \mathrm{ab}$ & 2.400 \\
\hline $40 \% \mathrm{ETV}$ & $9,9 \mathrm{~b}$ & 4,8 & $1.520 \mathrm{~b}$ & 2.433 \\
\hline Significancia & $*$ & n.s. & $* *$ & n.s. \\
\hline Interacción A x B & n.s. & n.s. & n.s & n.s \\
\hline
\end{tabular}

Valores con distinta letra en las columnas indican que hubo diferencias significativas de acuerdo a la prueba de comparación múltiple de Duncan $(\mathrm{p} \leq 0,05)$.

ETv: evapotranspiración real del viñedo; n.s.: no significativo; *: significativo $(\mathrm{p} \leq 0,05)$; **: altamente significativo (p $\leq 0,01)$.

\section{CONCLUSIONES}

En este estudio se encontró un efecto significativo de los tres niveles de poda $(12,18$, y 24 yemas por planta) y los tres niveles de reposición hídrica (100, 70 y $40 \%$ de la evapotranspiración real de la viñedo, ETV) sobre las características del follaje en ambas temporadas de estudio. Al respecto, un mayor porcentaje de racimos expuestos y menor número de capas de hojas fueron asociados a una mayor poda y menor reposición hídrica. En forma similar, el rendimiento se redujo significativamente a medida que aumentó la poda (menor número de yemas por planta) en ambas temporadas; sin embargo, sólo en la segunda temporada de estudio se observó un efecto significativo de la reposición hídrica.
En relación a la acidez total, $\mathrm{pH}$ y sólidos solubles, no se encontraron diferencias significativas entre los tratamientos de poda y reposición hídrica para ambas temporadas. Con respecto al diámetro de bayas, relación cutícula/pulpa, antocianas y polifenoles totales, no se observaron diferencias significativas entre los distintos niveles de poda ni de reposición hídrica durante la primera temporada de evaluación. Sin embargo, en la segunda temporada existieron diferencias significativas entre los tratamientos de poda y reposición hídrica sólo para las antocianas totales, observándose racimos con un mayor contenido de éstas en aquellos tratamientos con podas más severa.

Finalmente, en este estudio no existió un efecto significativo de la interacción entre la poda y la repo- 
sición hídrica sobre el crecimiento vegetativo, caracterización del follaje, componentes del rendimiento y composición de las bayas, excepto para el número de capas de hojas. En este caso, el mayor número de capas de hojas fue observado en el tratamiento con 24 yemas por planta y una reposición hídrica del $100 \%$ de la ETv.

\section{RECONOCIMIENTOS}

Esta investigación fue financiada por el proyecto FONDEF N ${ }^{\circ}$ D02I1045 y Viña San Pedro. También los autores desean agradecer a los ex-alumnos de la Escuela de Agronomía de la Universidad de Talca, ingenieros agrónomos, Sres. César Acevedo, Marcelo Duarte y Marcos Carrasco, por su participación en la colección de datos y mantención de la parcela experimental.

\section{LITERATURA CITADA}

Acevedo, C., S. Ortega-Farias, and Y. Moreno. 2004. Effect of three levels of water application during postsetting and post-veraison over vegetative development, productivity and grape quality on cv. Cabernet Sauvignon. Acta Hortic. 646:143-146.

Bergqvist, J., N.Dokoozlian, and N. Ebisuda. 2001. Sunlight exposure and temperature effects on berry growth and composition of Cabernet Sauvignon and Grenache in the Central San Joaquin Valley of California. Am. J. Enol. Vitic. 52:1-7.

Bravdo, B., Y. Hepner, C. Loinger, S. Cohen, and H. Tabacman. 1985. Effect of irrigation and crop level on growth, yield and wine quality of Cabernet Sauvignon. Am. J. Enol. Vitic. 36:132-139.

Choné, X., C. Van Leeuwen, P. Chéry, and P. RibéreauGayon. 2001. Terroir influence on water status and nitrogen status of non-irrigated Cabernet Sauvignon (Vitis vinifera). Vegetative development, must and wine composition (example of a Medoc top Estate vineyard, Saint Julien Area, Bordeaux, 1997). S. Afr. J. Enol. Vitic. 22:8-15.

Dry, P.R., and B.R. Loveys. 1999. Grapevine shoot growth and stomatal conductance are reduced when part of the root system is dried. Vitis 38:151-156.

Ferreyra, R., G. Sellés, J. Peralta, L. Burgos. y L. Valenzuela. 1998. Efecto del estrés hídrico aplicado en distintos períodos de desarrollo de la vid cv. Cabernet Sauvignon en la producción y calidad del vino. Santiago, Chile. Agric Téc. (Chile) 62:406-417.

Freeman, B.M. 1983. Effect of irrigation and pruning of Shiraz grapevines on subsequent red wine pigment. Am. J. Enol. Vitic. 34: 23-26.

Ginestar, C., J. Eastham, S. Gray, and P. Lland. 1998a. Use of sap flow sensor to schedule vineyard irrigation. I. Effect of post-veraison water deficit on water relations, vine growth, and yield of Shiraz grapevine. Am. J. Enol. Vitic. 49:413.
Ginestar, C., J. Eastham, S. Gray, and P. Lland. 1998b. Use of sap flow sensor to schedule vineyard irrigation. II. Effect of post-veraison water deficit on composition of Shiraz grapes. Am. J. Enol. Vitic. 49:421.

Goodwin, I.P. 1995. Irrigation of vineyard. A winegrape grower's guide to irrigation scheduling and regulated deficit irrigation. 56 p. Agriculture Victoria, Tatura, Australia.

Gurovich, L. 1998. Aplicación del riego deficitario controlado en la vid y el efecto sobre la calidad de la uva y el vino. p. 58-81. In P. Pszczólkowski y K. Sonneborn (eds). Tópicos de actualización en viticultura y enología. Colección de Extensión. Pontificia Universidad Católica, Facultad de Agronomía e Ingeniería Forestal, Santiago, Chile.

Hepner, Y., C. Bravdo, C. Loinger, S. Cohen, and H. Tabacman. 1985. Effect of drip irrigation schedules on growth, yield, must composition and wine quality of Cabernet Sauvignon. Am. J. Enol. Vitic. 36:77-83.

Kennedy, J., M. Matthews, and A.L.Waterhouse. 2002. Effect of maturity and vine water status on grape skin and wine flavonoids. Am. J. Enol. Vitic. 53:268-274.

Kliewer, W.M., G. Freeman, and C. Hossom. 1983. Effect of irrigation, crop level and potassium fertilization on Carignan vines. I. Degree of water stress and effect on growth and yield. Am. J. Enol. Vitic. 34:186-196.

Koundouras, S., C. Van Leeuwen, G. Seguin, et Y.Glories. 1997. Influence de l'alimentation en eau sur la croissance de la vigne, la maturation des raisins et les caractéristiques des vins en zone méditerranéenne (example de Némée, Gréce, cépage Saint-George, 1997). J. Int. Sci. Vigne Vin. 33:149-160.

Marquette, B. 1999. La madurez fenólica. Conceptos básicos. p. 25-49. In Seminario Internacional de Microbiología y Polifenoles del Vino. Universidad de Chile, Depto. de Agroindustria y Enología, Santiago, Chile. 
Miller, D.P., G.S. Howell, and J.A. Flore. 1996. Effect of shoot number on potted grapevines. I. Canopy development and morphology. Am. J. Enol. Vitic. 47:244-251.

Nadal, M., and L. Arola. 1995. Effects of limited irrigation on the composition of must and wine of Cabernet Sauvignon under semi-arid condition. Vitis 34:151-154.

Naor, A., B. Bravdo, and Y. Hepner. 1993. Effect of postveraison irrigation level on Sauvignon blanc yield, juice quality and water relations. S. Afr. J. Enol. Vitic. 14:19-25.

Ortega-Farias, S., M. Duarte, C. Acevedo, Y. Moreno, and F. Cordova. 2004a. Effect of four levels of water application on grape composition and midday stem water potencial on Vitis vinifera $\mathrm{L}$. cv. Cabernet Sauvignon. Acta Hort. (ISHS) 664:491-497.

Ortega-Farias, S., C. Acevedo, A. Acevedo, and B. Leyton. 2004b. Talca Irrigation Management System (TIMAS) for grapevine. Acta Hort. (ISHS) 664:499-504

Poni, S., A.N. Lakso, J.R. Turner, and R.E. Melious. 1994. The effect of pre- and post-veraison water on growth and physiology of potted Pinot Noir grapevines at crop level. Vitis 32:207-214.

Pszczólkowski, T., y E. Bordeu. 1984. Posibles causas del deterioro de la calidad del vino en parronales y viñedos vigorosos. Rev. Frut. (Chile) 5(1):23-26.

Reynier, A. 1989. Manual de viticultura. 382 p. $4^{\mathrm{a}}$ ed. Ediciones Mundi-Prensa, Madrid, España.
Sipiora, M., and M. Gutiérrez. 1998. Effect of preveraison irrigation cutoff and skin contact time on the composition, color and fenolic content of young Cabernet Sauvignon wine in Spain. Am. J. Enol. Vitic. Vol. 49:152-157.

Smart, R., and M. Robinson. 1991. Sunlight into wine. A handbook for wine-grape canopy management. $88 \mathrm{p}$. Winetitles, Adelaida, Australia.

Smart, R.E., and B.G. Coombe. 1983. Water relations of grapevines. p. 137-196. Vol. VII. In Kozlowski, T.T. (ed.). Water deficits and plant growth. Academic Press, New York, USA.

Smithyman, R., R. Wample, and N. Lang. 2001. Water deficit and crop level influences on photosynthetic strain and blackleaf symptom development in Concord grapevines. Am. J. Enol. Vitic. 52:364-375.

Tregoat, O., C. Van Leeuwen, X. Choné, et J.P. Gaudillere. 2002. Etude du régime hydrique et de la nutrition azotée de la vigne par des indicateurs physiologiques. Influence sur le comportement de la vigne et la maturation du raisin (Vitis vinifera L. cv. Merlot, 2000, Bordeaux). J. Int. Sci. Vigne Vin 36:133-142.

Williams, L.E., and M.A. Matthews. 1990. Grapevine. p. 1019-1055. In Stewart, B.A. and D.R. Nielsen (eds.). Irrigation of agricultural crops. Agronomy Series 30. American Society of Agronomy, Madison, Wisconsin, USA. 